\title{
Protein thermal stabilization in aqueous solutions of osmolytes
}

\author{
Piotr Bruździak ${ }^{1 凶}$, Aneta Panuszko', Muriel Jourdan² and Janusz Stangret ${ }^{1}$ \\ 1Department of Physical Chemistry, Chemical Faculty, Gdansk University of Technology, Gdańsk, Poland; ²Département de Chimie Moléculaire, \\ CNRS UMR5250, ICMG FR2607, Université de Grenoble, 570 Grenoble Cedex 9, France
}

Proteins' thermal stabilization is a significant problem in various biomedical, biotechnological, and technological applications. We investigated thermal stability of hen egg white lysozyme in aqueous solutions of the following stabilizing osmolytes: Glycine (GLY), N-methylglycine (NMG), N,N-dimethylglycine (DMG), N,N,N-trimethylglycine (TMG), and trimethyl-N-oxide (TMAO). Results of CD-UV spectroscopic investigation were compared with FTIR hydration studies' results. Selected osmolytes increased lysozyme's thermal stability in the following order: Gly $>$ NMG $>$ TMAO $\approx$ DMG $>$ TMG. Theoretical calculations (DFT) showed clearly that osmolytes' amino group protons and water molecules interacting with them played a distinctive role in protein thermal stabilization. The results brought us a step closer to the exact mechanism of protein stabilization by osmolytes.

Key words: water structure, protein stability, circular dichroism, FT-IR spectroscopy, DFT calculations

Received: 03 December, 2014; revised: 19 June, 2015; accepted: 22 September, 2015; available on-line: 23 October, 2015

\section{INTRODUCTION}

Osmolytes are small organic compounds accumulated in living cells in harsh environmental condition (Yancey, 2005; Yancey et al., 2004). Osmolytes' classification is difficult because of wide range of their chemical origin and composition. They possess an extra feature - they can modify macromolecules' stability. This influence is usually nonspecific, i.e. stabilizing properties are universal in a given group of macromolecules. However, a good protein stabilizer can also be a strong nucleic acid denaturant (e.g. Glycine Betaine) or can change its stabilizing/destabilizing abilities in a different protonation state (Singh et al., 2011, Denning et al., 2013). Osmolytes may influence the energetic barrier between native and intermediate states during protein denaturation, and delay or promote unfavorable aggregation processes (Seeliger et al., 2013; Wei et al., 2013). The exact stabilization or destabilization driving force is still under debate. Many theories were proposed to explain the basics of osmolytes' influence on protein stability (Auton et al., 2011; Mondal et al., 2013; Damodaran, 2012; Bennion \& Daggett, 2003). However, none of them explained it sufficiently, although the insight into the subject of protein stability and stabilization mechanism is of the great importance from the practical and scientific points of view.

Protein thermal stability is a superposition of thermodynamic stability (characterized by $\Delta G$ of the denaturation process) and kinetic stability (dependent on the barrier Gibbs' function $\Delta G^{t}$ between native and denatured states). Water molecules affect proteins' structure in solution both through hydrophobic effect and by participation in favorable interactions at the protein surface. Osmolytes' presence can interfere in both of these phenomena. Mostly accepted hypothesis concerning the basis of osmolytes' stabilizing and destabilizing effects are focused on their influence on a biomolecule's denatured state. There is a general consensus that osmolytes interact favorably with protein's unfolded form through direct hydrogen bonds. This interaction causes the enthalpic stabilization of the unfolded state because the number of peptide groups available to this kind of osmolyte is higher than in the native form of a protein.

In the case of stabilizing osmolytes (e.g. aminoacids and their derivatives) it is stated that they are preferentially excluded from the protein surface. Therefore, the presence of these osmolytes should destabilize the unfolded state through the decrease of effective volume available to random coil (as the effect of conformational entropy decrease). In this kind of considerations, stabilizing and destabilizing osmolytes' influence on protein's native state is usually ignored.

Our results (Panuszko et al., 2009; Bruździak et al., 2013; Panuszko et al., 2012) made us lean toward the hypothesis that osmolytes' influence on proteins is determined by their ability to modify structural and dynamic properties of water in protein hydration shell. Stabilization/destabilization of protein native state may have an influence on $\Delta G$ of denaturation process, as well as on the transition state barrier height of the unfolding process.

Our previous studies concerning hen egg white lysozyme's denaturation (Panuszko et al., 2009; Bruździak et al., 2013; Panuszko et al., 2012) allowed us to suspect that no direct interactions occur between lysozyme and osmolytes, and that water properties are the most important factors affecting the stability of protein: (1) lysozyme's secondary structure does not change significantly in the presence of many osmolytes studied so far (Panuszko et al., 2009; Bruździak et al., 2013); (2) a clear difference exists between the influence of some stabilizing and destabilizing osmolytes on organization of water molecules surrounding them (Panuszko et al., 2012); (3) energetic and structural properties of water affected by stabilizing osmolytes are significantly similar to the properties of water affected by lysozyme in its native state (Bruździak et al., 2013); (4) strong interrelationships exists between water properties in osmolytes' hydration spheres and their influence on lysozyme thermal stability

e-mail: piotr.bruzdziak@pg.gda.p

Abbreviations: CD-UV, circular dichroism spectroscopy; DFT, density functional theory; DSC, differential scanning calorimetry; FTIR, Fourier transform infrared spectroscopy; NMG, N-methylglycine; DMG, $N, N$-dimethylglycine; TMAO, trimethyl- $N$-oxide; TMG, $N, N, N$ trimethylglycine 
(Bruździak et al., 2013). In all these cases water properties characterized by the distance distribution function (obtained from infrared spectral data) between oxygenoxygen atoms of water molecules which are influenced by osmolytes or by lysozyme.

We used circular dichroism in the UV region (CDUV) and performed DFT calculations of aqueous clusters of the solutes using the polarized continuum model (PCM). Osmolytes selected for this paper are generally considered as protein stabilizers: Glycine, $N$-methylglycin (NMG, Sarcosine), N,N-dimethylglycine (DMG), N,N,Ntrimethylglycine (TMG, Betaine), and trimethyl-N-oxide (TMAO). TMAO is considered as one of the strongest stabilizers in nature, and participate in the protection of renal cells of mammals from deleterious influence of urea (Jackson-Atogi et al., 2013; Sarma \& Paul, 2013), or stabilization of the cells of deep see organisms against high pressure (Sarma \& Paul, 2013; Sarma \& Paul, 2012). Glycine, NMG, DMG, and TMG (Betaine) are generally considered as moderate stabilizers (Santoro et al., 1992; Wang et al., 2013; Guinn et al., 2011; Caldas et al., 1999).

\section{MATERIAL AND METHODS}

HEW lysozyme (Fluka) was dissolved in deionized water (about $3 \mathrm{~g} / 15 \mathrm{~mL}$ ) and dialyzed against water $\left(24 \mathrm{~h} / 4^{\circ} \mathrm{C}\right.$, with membrane cut-off $\left.10 \mathrm{kDa}\right)$. The dialyzed solution was then lyophilized $\left(10 \mathrm{~mm} \mathrm{Hg} /-60^{\circ} \mathrm{C} /\right.$ $48 \mathrm{~h}$ ). The lysozyme native secondary structure was restored after re-solvation in water (determined with FTIR spectroscopy). All osmolytes: Glycine (Sigma), NMG (Aldrich), DMG (Sigma), TMG (Sigma), TMAO (Fluka) were used as supplied. All the osmolytes solutions were prepared in $20 \mathrm{mM}$ phosphate buffer, $\mathrm{pH}$ 6.5. These solutions were used to dissolve appropriate amounts of purified protein to a final concentration of $0.4 \mathrm{mg} / \mathrm{mL}$ $\left(0.028 \mathrm{~mol} / \mathrm{dm}^{3}\right)$.

All spectra and denaturation curves were recorded using Jasco J-810 CD-UV spectrometer, equipped with Peltier temperature controller. For each sample a spectrum at $25^{\circ} \mathrm{C}$ before denaturation was measured. All spectra were recorded with $0.5 \mathrm{~nm}$ resolution, and a bandwidth of $4 \mathrm{~nm}$, and three repeats were recorded and averaged. The temperature scanning speed was set to $0.5^{\circ} \mathrm{C} / \mathrm{min}$. The spectra were baseline corrected by solvent subtraction. In almost every sample osmolytes severely deteriorated the quality of the spectra in the low wavenumber region. Thus, we were unable to measure full CD-UV spectra of lysozyme in the presence of osmolytes, and all spectra were recorded between 275-220 nm.

Denaturation curves were measured from $65^{\circ} \mathrm{C}$ to $85^{\circ} \mathrm{C}$, with a $0.1^{\circ} \mathrm{C}$ temperature step. The delay before recording the signal was set up to $60 \mathrm{~s}$. In each case the wavelength of denaturation curve measurement was set up to $225 \mathrm{~nm}$. Only in the case of two solutions of TMG (1.00 and $\left.1.50 \mathrm{~mol} / \mathrm{dm}^{3}\right)$ denaturation curves were measured at $230 \mathrm{~nm}$ because of noisy data at $225 \mathrm{~nm}$. This did not affect the melting temperature, because the denaturation of lysozyme is a co-operative process, as determined previously in our DSC studies (Bruździak et al., 2013). Denaturation temperatures of lysozyme in the presence of osmolytes were determined using the maximum of the first derivative of denaturation curves (Savitsky-Golay algorithm with $5^{\circ} \mathrm{C}$ window and third-order polynomial). The maximum of the derivative is a more straightforward method of $T_{\mathrm{m}}$ determination and does not require a model fitting step. To compare osmolytes' influence on lysozyme thermal stability we proposed to use a $\left(d T_{\mathrm{m}} / d C\right)_{C \rightarrow 0}$ parameter, which is the derivative of the curve at the zero molar concentration showing the denaturation temperature evolution as the osmolyte concentration function (Bruździak et al., 2013). The propagation of $\left(d T_{\mathrm{m}} / d C\right)_{C \rightarrow 0}$ values errors was estimated on the basis of $N$ error $( \pm 0.5$, determined by Panuszko et al., 2011) and the intercept errors of the fitted quadratic curves from Fig. 1.

DFT calculations were performed using GAUSSIAN 03 package (Frish et al., 2003). The optimized geometries and energies of the non-hydrated and hydrated structures of osmolytes were calculated using density functional theory (DFT) with B3LYP hybrid functional using the $6-311++\mathrm{G}(\mathrm{d}, \mathrm{p})$ basis set (Krishnan et al., 1980). The polarizable continuum model (PCM) formalism of the self-consistent reaction field theory (SCRF) was used to study systems in the liquid phase (Cossi et al., 2002; Mennucci \& Tomasi, 1997; Tomasi et al., 1999). Initial structures of all studied systems showed C1 symmetry and no symmetry constraints were imposed during geometry optimizations. Water molecules were placed near an appropriate osmolyte molecule atom so that the intermolecular distance between hydrogen of water molecule and osmolyte's oxygen atom or between oxygen atom of water molecule and osmolyte's amino proton had length of about $1.45 \AA$ to force hydrogen bond formation. Additionally, individual spheres were placed on each of the osmolyte hydrogen atoms (explicit hydrogens) to assure the continuity of the PCM cavity. The zero point energy contribution to the vibrational energy was taken into account. Covalent contributions to the hydrogen-bond energy for the complexes were calculated as the differences between the total energies of the complexes (in the water continuum) and the energies of isolated monomers (in the water continuum), and have been corrected for the basis set superposition error (BSSE) using the standard counterpoise method (Boys \& Bernardi,1970). The covalent energy effect is weak for weak H-bonds, but it increases distinctly as the H-bond energy increases (Grabowski et al., 2006). HyperChem 8 software was used at the stage of visualization of computed results.

\section{RESULTS AND DISCUSSION}

\section{Osmolytes influence on lysozyme thermal stability}

All of the data concerning osmolytes' stabilizing effect on the lysozyme denaturation temperature are presented in Table 1. The dependencies of lysozyme's melting temperature vs. osmolyte's concentration are shown in Fig. 1. Glycine appears to be the most effective stabilizer among all other selected osmolytes in CD-UV experimental conditions. The $\left(d T_{\mathrm{m}} / d C\right)_{C \rightarrow 0}$ parameter calculated for this compound is very high $\left(8.2 \mathrm{~K} \cdot \mathrm{dm}^{3} / \mathrm{mol}\right)$ (Table 2). Its mono- and dimethyl derivatives (NMG and DMG) influence the lysozyme melting temperature in similar way, with lower $\left(d T_{\mathrm{m}} / d C\right)_{C \rightarrow 0}$ parameters: 6.6 and $5.5 \mathrm{~K} \cdot \mathrm{dm}^{3} / \mathrm{mol}$, respectively. In this group of compounds TMG is the less effective stabilizer, with $\left(d T_{\mathrm{m}} /\right.$ $d C)_{C \rightarrow 0}$ parameter equal to $2.7 \mathrm{~K} \cdot \mathrm{dm}^{3} / \mathrm{mol}$. Surprisingly, TMAO, which is considered as one of the most effective protein stabilizers, exhibits $\left(d T_{\mathrm{m}} / d C\right)_{C \rightarrow 0}$ parameter (5.7 $\mathrm{K} \cdot \mathrm{dm}^{3} / \mathrm{mol}$ ) lower than glycine, its methyl derivatve, and close to dimethyl derivatives.

Melting temperatures measured in the present study by $\mathrm{CD}-\mathrm{UV}$ are lower by 2 to $3^{\circ} \mathrm{C}$ than previously reported ones obtained by the DSC method and corresponding to a higher protein concentration (Bruździak et 
Table 1. Parameters of denaturation of lysozyme in the presence of stabilizing osmolytes.

\begin{tabular}{|c|c|c|c|c|c|}
\hline \multicolumn{2}{|c|}{ GLY } & \multicolumn{2}{|c|}{ NMG } & \multicolumn{2}{|c|}{ DMG } \\
\hline $\mathrm{Ca}^{\mathrm{a}}$ & $T_{\mathrm{m}}^{\mathrm{b}}$ & $\mathrm{Ca}^{\mathrm{a}}$ & $T_{\mathrm{m}}^{\mathrm{b}}$ & $\mathrm{Ca}^{\mathrm{a}}$ & $T_{\mathrm{m}}^{\mathrm{b}}$ \\
\hline $\mathrm{mol} \cdot \mathrm{dm}^{-3}$ & ${ }^{\circ} \mathrm{C}$ & $\mathrm{mol} \cdot \mathrm{dm}^{-3}$ & ${ }^{\circ} \mathrm{C}$ & $\mathrm{mol} \cdot \mathrm{dm}^{-3}$ & ${ }^{\circ} \mathrm{C}$ \\
\hline 0.50 & $75.8 \pm 0.6$ & 0.49 & $75.1 \pm 0.3$ & 0.49 & $74.8 \pm 0.4$ \\
\hline 1.00 & $78.7 \pm 0.5$ & 0.97 & $77.8 \pm 0.3$ & 0.97 & $77.4 \pm 0.5$ \\
\hline 1.50 & $80.7 \pm 0.6$ & 1.45 & $80.1 \pm 0.3$ & 1.46 & $80.2 \pm 0.5$ \\
\hline \multicolumn{2}{|c|}{ TMG } & \multicolumn{2}{|c|}{ TMAO } & \multicolumn{2}{|c|}{ Lysozyme } \\
\hline $\mathrm{Ca}^{\mathrm{a}}$ & $T_{\mathrm{m}}^{\mathrm{b}}$ & $\mathrm{Ca}^{\mathrm{a}}$ & $T_{\mathrm{m}}^{\mathrm{b}}$ & $\mathrm{Ca}^{\mathrm{a}}$ & $T_{\mathrm{m}}^{\mathrm{b}}$ \\
\hline $\mathrm{mol} \cdot \mathrm{dm}^{-3}$ & ${ }^{\circ} \mathrm{C}$ & $\mathrm{mol} \cdot \mathrm{dm}^{-3}$ & ${ }^{\circ} \mathrm{C}$ & $\mathrm{mol} \cdot \mathrm{dm}^{-3}$ & ${ }^{\circ} \mathrm{C}$ \\
\hline 0.50 & $73.1 \pm 0.5$ & 0.43 & $74.1 \pm 0.5$ & 0.00 & $72.1 \pm 0.4$ \\
\hline 1.00 & $74.4 \pm 0.4$ & 0.87 & $76.5 \pm 0.5$ & & \\
\hline 1.50 & $74.9 \pm 0.4$ & 1.31 & $77.5 \pm 0.5$ & & \\
\hline
\end{tabular}

a Osmolyte concentration. ${ }^{b}$ Melting temperature (maximum of the first derivative of the denaturation curve, uncertainties result from the melting curve noisiness).

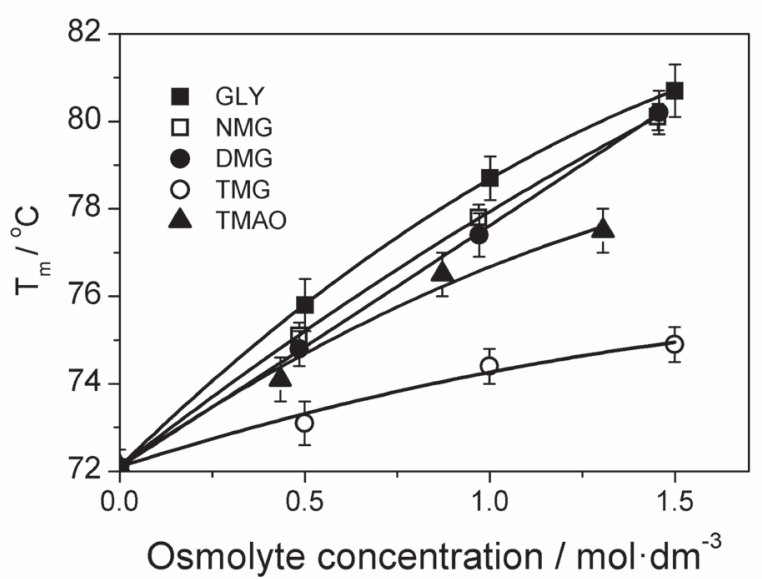

Figure 1. The dependencies of lysozyme's denaturation temperature (the maximum of the first derivative of CD-UV melting curves) versus osmolytes concentration.

al., 2013). The higher protein concentration might have promoted protein self-stabilization and thus increase the observed melting temperature. Thus, the order of stabilizers obtained from the CD-UV melting curves is as follows: $\mathrm{Gly}>\mathrm{NMG}>\mathrm{TMAO} \approx \mathrm{DMG}>\mathrm{TMG}$. This stabilizers' order correlates quite well with the number of water molecules $(N)$ affected by these compounds that was previously determined by FTIR studies (see Table 2), except TMAO which affects a large number of water

Table 2. The influence of stabilizing osmolytes on lysozyme thermal stability in respect to their influence on water molecules

\begin{tabular}{lccc}
\hline & $\mathrm{N}^{\mathrm{a}}$ & $d T_{\mathrm{m}} / \mathrm{dC}$ & $\left(d T_{\mathrm{m}} / \mathrm{dC}\right) / \mathrm{N}$ \\
\hline & & $\mathrm{K} \cdot \mathrm{mol}^{-1} \cdot \mathrm{dm}^{3}$ & $\mathrm{~K} \cdot \mathrm{mol}^{-1} \cdot \mathrm{dm}^{3}$ \\
\hline GLY & $5.8 \pm 0.5$ & $8.2 \pm 1.2$ & $1.4 \pm 0.3$ \\
\hline NMG & $5.0 \pm 0.5$ & $6.6 \pm 0.7$ & $1.3 \pm 0.3$ \\
\hline DMG & $4.8 \pm 0.5$ & $5.5 \pm 1.0$ & $1.1 \pm 0.3$ \\
\hline TMG & $4.0 \pm 0.5$ & $2.7 \pm 1.0$ & $0.7 \pm 0.3$ \\
\hline TMAO & $8.5 \pm 0.5$ & $5.7 \pm 1.3$ & $0.7 \pm 0.2$ \\
\hline
\end{tabular}

${ }^{a}$ The number of water molecules affected by osmolytes. molecules $(N=8.5)$. However, after recalculation of the $\left(d T_{\mathrm{m}} / d C\right)_{C \rightarrow 0}$ parameter per one affected water molecule (i.e. to obtain the contribution of a single affected water molecule in the $T_{\mathrm{m}}$ increase $-\left(d T_{\mathrm{m}} / d C\right)_{C \rightarrow 0} / N$ parameter, see Table 2) one can see that osmolytes can be divided into two subgroups: Gly-NMG-DMG (possessing amino protons), and TMG-TMAO (possessing no amino protons). On the other hand, it can be seen that CD-UV and previous DSC results are similar, and both correlate well with the previously obtained most probable intermolecular oxygen-oxygen $\left(R_{O O}{ }^{\circ}\right)$ distance of water molecules affected by osmolyte (Panuszko et al., 2009; Bruździak et al., 2013; Panuszko et al., 2011). This observation proves that the interdependence of water properties in osmolytes' hydration spheres and their influence on lysozyme thermal stability exists.

\section{Amino protons promote osmolyte's stabilizing property}

The DFT calculations of stabilizing osmolytes in the aqueous solution (PCM model) gives an insight into the observed results and correlations between CD-UV, and FTIR data. The PCM model was used because only in the PCM model their stable form is the zwitterion (as in real solution). The procedure used in this case takes into account only the covalent contribution of hydrogen bond energies. Nonspecific interactions like dipole-dipole, dipole-induced dipole, and dispersive forces, are hidden in the values of water cluster energies, separated central molecule (i.e. separated osmolyte molecule), and separated water molecule in the PCM model. Direct interactions between water molecules and different hydrophilic centers of osmolytes were analyzed. We examined complexes in which osmolytes' hydrophilic centers formed an utmost number of direct hydrogen bonds. All considered structures are presented in Fig. 2. On the basis of the difference between the water cluster energy value and the sum of separated water molecules energies we calculated the hydrogen bond energies' covalent contributions. Next, the obtained value was divided by the water molecule number in each analyzed structure. It is believed that the obtained value corresponds to the hydrogen bond energy's covalent contribution in respect to one water molecule. These results are presented in Table 3, while Fig. 3 shows the average energetic characteristics of hydrogen bonds covalent contribution (in 
A

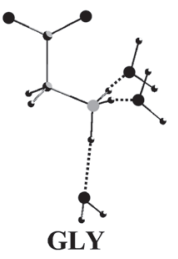<smiles>C=C(C)C(C(C)C)C(C)C(C)C</smiles>

NMG
B

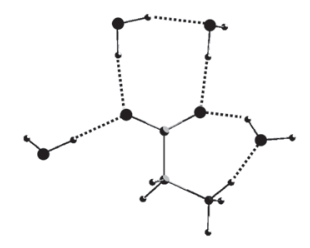

GLY

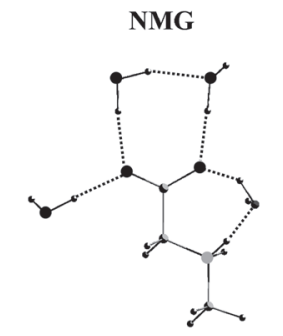

NMG<smiles>CC(C)C(C)C(C)(C(C)C)C(C)C</smiles>

DMG

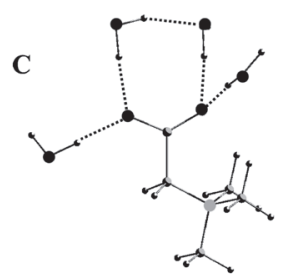

TMG

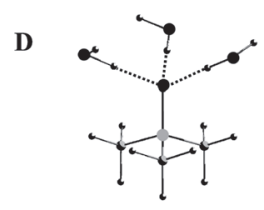

TMAO
Figure 2. Hydrogen bonds formed between hydrophilic groups of osmolytes and water molecules.

Larger black spheres denote oxygen atoms; larger grey spheres denote nitrogen atoms; small gray spheres denote hydrogen atoms. The hydrogen bonds are indicated with dashed lines. Water complexes for glycine are taken from Bruździak et al., 2013.

Table 3. Total energies, covalent contributions to the hydrogenbond energies and covalent contributions to the hydrogen-bond energies per one water molecule of osmolyte water clusters calculated at the B3LYP/6-311++G(d,p) level of theory in the PCM model

\begin{tabular}{|c|c|c|c|c|}
\hline & & $\mathrm{E}^{\mathrm{a}}$ & $\mathrm{E}_{\mathrm{H} \text {-bond }}{ }^{\mathrm{b}}$ & $\mathrm{E}_{\mathrm{H} \text {-bond } / \mathrm{nH} 2 \mathrm{O}^{\mathrm{c}}}$ \\
\hline & $\mathrm{H}_{2} \mathrm{O}$ & -76.452346 & & \\
\hline & GLYd & -284.471558 & & \\
\hline A & $\mathrm{GLY}+3 \mathrm{H}_{2} \mathrm{O}$ & -513.840305 & -17.44 & -5.81 \\
\hline \multirow[t]{2}{*}{ B } & $\mathrm{GLY}+4 \mathrm{H}_{2} \mathrm{O}$ & -590.291549 & -13.70 & -3.43 \\
\hline & NMG & -323.758991 & & \\
\hline A & $\mathrm{NMG}+2 \mathrm{H}_{2} \mathrm{O}$ & -476.670056 & -7.06 & -3.53 \\
\hline \multirow[t]{2}{*}{ B } & $\mathrm{NMG}+4 \mathrm{H}_{2} \mathrm{O}$ & -629.576805 & -7.88 & -1.97 \\
\hline & DMG & -363.043537 & & \\
\hline A & $\mathrm{DMG}+\mathrm{H}_{2} \mathrm{O}$ & -439.498062 & -0.73 & -0.73 \\
\hline \multirow[t]{2}{*}{ B } & $\mathrm{DMG}+4 \mathrm{H}_{2} \mathrm{O}$ & -668.858263 & +0.56 & +0.14 \\
\hline & TMG & -402.321585 & & \\
\hline \multirow[t]{2}{*}{ C } & $\mathrm{TMG}+4 \mathrm{H}_{2} \mathrm{O}$ & -708.139341 & -8.83 & -2.21 \\
\hline & $\mathrm{TMAO}^{\mathrm{d}}$ & -249.597040 & & \\
\hline D & $\mathrm{TMAO}+3 \mathrm{H}_{2} \mathrm{O}$ & -478.961047 & -8.83 & -2.94 \\
\hline
\end{tabular}

aTotal energy (hartree). ${ }^{b}$ Covalent contribution to the hydrogen-bond energy calculated as the difference between the energy of a cluster in the water continuum and the sum of the energies of separated molecules in the water continuum $\left(\mathrm{kJ} \cdot \mathrm{mol}^{-1}\right)$. cCovalent contribution to the hydrogen-bond energy per one water molecule $\left(\mathrm{kJ} \cdot \mathrm{mol}^{-1}\right)$. dData are taken from Ref. 26 and Ref. 25, respectively. All the covalent contributions to the hydrogen-bond energy were corrected for the basis set superposition error (BSSE), using counterpoise procedure. The letters $A$, $B, C$ and $D$ refer to respective labels in Fig. 2 . group.

The DFT calculations of osmolytes' water clusters drew us to the conclusion that lysozyme's higher thermal stability is assured by an osmolyte molecule possessing a higher number of hydrogen atoms on the amino group. It appears that the strong interaction of water molecules with osmolyte's amino groups determines osmolyte's influence on protein thermal stability. This

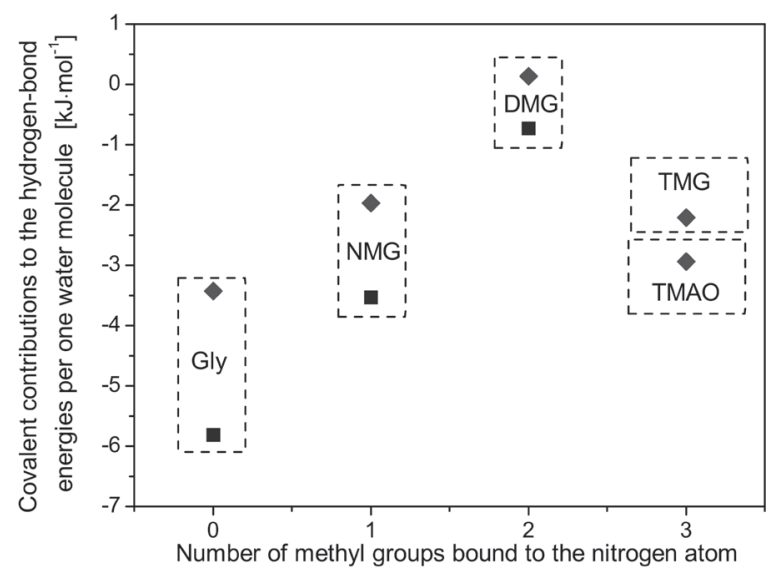

Figure 3. Energetic characteristics of covalent share of hydrogen bonds (in respect to one water molecule) between water molecules and hydrophilic centers of osmolytes:

square denotes interaction between water molecules and amine group, rhombus denotes interaction between water molecules and carboxylic group (or oxygen atom, in the case of TMAO). All values correspond to complexes presented in Fig. 2. 
can be explained by taking into account our previous observation concerning lysozyme hydration (Panuszko et al., 2012). The infrared spectrum of lysozyme hydration water may be treated as a superposition of two absorption contributions: water molecules affected by $N$ methylacetamide (NMA) and by carboxylate anion. We also noticed that water molecules were not involved in the H-bond formation with the amino group of NMA molecule (Panuszko et al., 2008). Therefore, water molecules strongly $\mathrm{H}$-bonded by oxygen atom to the amino group of currently studied osmolytes can form stronger H-bonds (because of the cooperative effect) by hydrogen atoms with above mentioned oxygen centers on the surface of lysozyme. According to our previous experimental FTIR results stabilizing osmolytes do not interact directly with the protein. Thus, we can conclude that it is the osmolyte affected water that influences protein directly, not the osmolyte molecule itself.

Osmolyte's ability to stabilize protein decreases with the number of methyl substituents: Gly $>$ NMG $>$ DMG. TMG and TMAO, which do not possess amino hydrogens, turned out to be weaker stabilizers. In this case, the interaction between water molecule and carboxylic group is the parameter determining their stabilizing ability. Additionally, the higher value of covalent hydrogen bond energy contribution indicates better stabilizing ability (i.e. TMAO is a better stabilizer than TMG). These results are in agreement with CD-UV melting curves, and FTIR data.

\section{CONCLUSIONS}

The correlation between CD-UV data and previously published results concerning water structure around osmolyte molecules ( $N$ parameters) allowed us to divide the investigated group of stabilizing osmolytes into two groups: good stabilizers (Gly, NMG, DMG with ( $d T_{\mathrm{m}}$ / $d C)_{C \rightarrow 0} / N$ between 1.4 and 1.1) and weak stabilizers (TMG and TMAO with $\left.\left(d T_{\mathrm{m}} / d C\right)_{C \rightarrow 0} / N \sim 0.7\right)$. These results are also supported and explained by DFT calculations - molecules possessing amino protons are better stabilizers. We suspect that the interaction of water molecules with these protons is responsible for osmolytes' ability to protein thermal stabilization. The ability decreases with the number of methyl substituents on their amino groups, though the decrease is small.

It appears that water molecule interacting with osmolyte's amino proton, which can act as a proton donor in protein-water interactions, possesses structural and energetic characteristics allowing to interact with proteins in a favorable manner. In the case of osmolytes possessing no amino protons, stabilizing properties of an osmolyte increase with the strength of water-osmolyte interaction (through the oxygen atom).

\section{Acknowledgements}

This study was supported by a grant from the $\mathrm{Na}$ tional Science Center of the Republic of Poland No. 2013/11/B/NZ1/02258).

We would like to thank Jean-François Constant for his support and help during preparation of the manuscript.

\section{REFERENCES}

Auton M, Roesgen J, Sinev M, Holthauzen LMF, Bolen DW (2011) Osmolyte effects on protein stability and solubility: A balancing act between backbone and side-chains. Biophys Chem 159: 90-99. http:// dx.doi.org/10.1016/j.bpc.2011.05.012.
Bennion BJ, Daggett V (2003) The molecular basis for the chemical denaturation of proteins by urea. Proc Natl Acad Sci USA 100: 5142-5147. http://dx.doi.org/10.1073/pnas.0930122100.

Boys SF, Bernardi F (1970) The calculation of small molecular interactions by differences of separate total energies. Some procedures with reduces errors. Mol Phys 19: 553-566. http://dx.doi. org/10.1080/00268977000101561.

Bruździak P, Panuszko A, Stangret J (2013) Influence of osmolytes on protein and water structure: a step to understanding the mechanism of protein stabilization. J Phys Chem B 117: 11502-11508. http:// dx.doi.org/10.1021/jp404780c.

Caldas T, Demont-Caulet N, Ghazi A, Richarme G (1999) Thermoprotection by glycine betaine and choline. Microbiol-Uk 145: $2543-$ 2548. http://dx.doi.org/10.1099/00221287-145-9-2543.

Cossi M, Scalmani G, Rega N, Barone V (2002) New developments in the polarizable continuum model for quantum mechanical and classical calculations on molecules in solution. J Chem Phys 117: 43-54. http://dx.doi.org/10.1063/1.1480445.

Damodaran S (2012) On the molecular mechanism of stabilization of proteins by cosolvents: role of Lifshitz electrodynamic forces. Langmuir 28: 9475-9486. http://dx.doi.org/10.1021/la300953n.

Denning EJ, Thirumalai D, MacKerell AD (2013) Protonation of trimethylamine $\mathrm{N}$-oxide (TMAO) is required for stabilization of RNA tertiary structure. Biophys Chem 184: 8-16. http://dx.doi. org/10.1016/j.bpc.2013.08.002.

Frisch M et al. (2003) Gaussian 03, Revision C.02.

Grabowski SJ, Sokalski WA, Dyguda W, Leszczyński J (2006) Quantitative classification of covalent and noncovalent H-bonds. $J$ Phys Chem B 110: 6444-6446. http://dx.doi.org/10.1021/jp0600817.

Guinn EJ, Pegram LM, Capp MW, Pollock MN, Record MT (2011) Quantifying why urea is a protein denaturant, whereas glycine betaine is a protein stabilizer. Proc Natl Acad Sci USA 108: 16932_ 16937. http://dx.doi.org/10.1073/pnas.1109372108.

Jackson-Atogi R, Sinha PK, Roesgen J (2013) Distinctive solvation patterns make renal osmolytes diverse. Biophys J 105: 2166-2174. http://dx.doi.org/10.1016/j.bpj.2013.09.019.

Krishnan R, Binkley J, Seeger R, Pople J (1980) Self-consistent molecular-orbital methods. XX. Basis set for correlated wave-functions. I Chem Phys 72: 650-654. http://dx.doi.org/10.1063/1.438955.

Mennucci B, Tomasi J (1997) Continuum solvation models: A new approach to the problem of solute's charge distribution and cavity boundaries. I Chem Phys 106: 5151-5158. http://dx.doi. org $/ 10.1063 / 1.473558$

Mondal J, Stirnemann G, Berne BJ (2013) When does trimethylamine $\mathrm{N}$-oxide fold a polymer chain and urea unfold it? I Phys Chem B 117: 8723-8732. http://dx.doi.org/10.1021/jp405609j.

Panuszko A, Bruździak P, Zielkiewicz J, Wyrzykowski D, Stangret J (2009) Effects of urea and trimethylamine-N-oxide on the properties of water and the secondary structure of hen egg white lysozyme. J Phys Chem B 113: 14797-14809. http://dx.doi.org/10.1021/ jp904001m.

Panuszko A, Gojło E, Zielkiewicz J, Śmiechowski M, Krakowiak J, Stangret J (2008) Hydration of simple amides. FTIR spectra of HDO and theoretical studies. J Phys Chem B 112: 2483-2493. http:// dx.doi.org/10.1021/jp7099509.

Panuszko A, Śmiechowski M, Stangret J (2011) Fourier transform infrared spectroscopic and theoretical study of water interactions with glycine and its N-methylated derivatives. J Chem Phys 134: 115104. http://dx.doi.org/10.1063/1.3567202.

Panuszko A, Wojciechowski M, Bruzdziak P, Rakowska PW, Stangret J (2012) Characteristics of hydration water around hen egg lysozyme as the protein model in aqueous solution. FTIR spectroscopy and molecular dynamics simulation. Phys Chem Chem Phys 14: 1576515773. http://dx.doi.org/10.1039/c2cp42229h.

Santoro M, Liu Y, Khan S, Hou L, Bolen D (1992) Increased thermalstability of proteins in the presence of naturally-occurring osmolytes. Biochemstry 31: 5278-5283. http://dx.doi.org/10.1021/bi00138a006.

Sarma R, Paul S (2012) The effect of aqueous solutions of trimethylamine-N-oxide on pressure induced modifications of hydrophobic interactions. I Chem Phys 137: 094502. http://dx.doi. org/10.1063/1.4748101.

Sarma R, Paul S (2013) Exploring the molecular mechanism of trimethylamine-N-oxide's ability to counteract the protein denaturing effects of urea. I Phys Chem B 117: 5691-5704. http://dx.doi. org/10.1021/jp401750v.

Seeliger J, Estel K, Erwin N, Winter R (2013) Cosolvent effects on the fibrillation reaction of human IAPP. Phys Chem Chem Phys 15: 8902-8907. http://dx.doi.org/10.1039/c3cp44412k.

Singh LR, Poddar NK, Dar TA, Kumar R, Ahmad F (2011) Protein and DNA destabilization by osmolytes: The other side of the coin. Life Sci 88: 117-125. http://dx.doi.org/10.1016/j.lfs.2010.10.020.

Tomasi J, Mennucci B, Cancès E (1999) The IEF version of the PCM solvation method: an overview of a new method addressed to study molecular solutes at the QM ab initio level. I Mol Struct THEOCHEM 464: 211-226. http://dx.doi.org/10.1016/S01661280(98)00553-3. 
Wang W, Lee J, Jin Q-X, Fang N-Y, Si Y-X, Yin S-J, Qian G-Y, Park Y-D (2013) Effects of osmolytes on pelodiscus sinensis creatine kinase: a study on thermal denaturation and aggregation. Int J Biol Macromol 60: 277-287. http://dx.doi.org/10.1016/j.ijbiomac.2013.06.015.

Wei M, Hai P, Meng Q, Yi C, Wei W (2013) Osmolyte effects on the unfolding pathway of beta-lactoglobulin. Chin Phys Lett 20: 108701.
Yancey PH (2005) Organic osmolytes as compatible, metabolic and counteracting cytoprotectants in high osmolarity and other stresses. J Exp Biol 208: 2819-2830. http://dx.doi.org/10.1242/jeb.01730.

Yancey PH, Rhea MD, Kemp KM, Bailey DM (2004) Trimethylamine oxide, betaine and other osmolytes in deep-sea animals: Depth trends and effects on enzymes under hydrostatic pressure. Cell Mol Biol 50: 371-376. http://dx.doi.org/10.1170/T526. 